\section{A Universal view}

\author{
Higher Than Everest: An \\ Adventurer's Guide to \\ the Solar System \\ by Paul Hodge \\ Cambridge University Press: 2001. 256 pp. \\ $\mathfrak{1} 18.95, \$ 27.95$
}

Solar System Evolution: A New

Perspective. 2nd edition

by Stuart Ross Taylor

Cambridge University Press: 2001. 484 pp.

$\mathfrak{E} 60, \$ 90$

\section{John E. Chambers}

The Solar System is a truly remarkable place, and well worth a visit. This, at least, is the shared conclusion of two new books on the subject. But there the similarities end. Higher Than Everest is a light-hearted adventurer's guide to our planet's immediate neighbourhood, whereas Stuart Ross Taylor's book is an altogether weightier tome with a loftier goal in mind - to provide a scientific history of the Solar System.

Higher Than Everest is the kind of book that will appeal to armchair alpinists everywhere, and one that sets the standard for twenty-second-century aficionados of extreme sports. The book opens with an account of a hypothetical expedition to climb Olympus Mons, the tallest volcano in the Solar System that dwarfs all of the 'seven summits' here on Earth. Although the ascent is not difficult from a climber's point of view, would-be Reinhold Messners face a number of hurdles en route before they can bag the summit.

Paul Hodge provides a detailed plan of campaign with a generous amount of time set aside for scientific exploration along the way. This approach sets the tone for the rest of the book. As he moves to Venus, Io, Miranda and beyond, Hodge seizes every opportunity to insert snippets of hard science into his description of each breathtaking piece of scenery. Jargon is kept to a minimum, and the emphasis is on the 'what' rather than the 'why', but Hodge nonetheless manages to address a number of issues at the cutting edge of planetary science. Compared with Mars, journeys to the outer Solar System are technically challenging and fraught with uncertainty. But despite this, Hodge leaves the reader feeling that one day they might just happen.

Since the publication of the first edition of Solar System Evolution a decade ago, planetary science has advanced by leaps and bounds. The Edgeworth-Kuiper belt has been exposed (literally), the Galileo mission and comet Shoemaker-Levy 9 have transformed our view of the jovian system, and extrasolar planets - those beyond the Solar
System - are now an established fact. Yet despite these advances, understanding the origins and modern characteristics of the Solar System remains a frustratingly difficult task. This fully updated survey of the field is a noble attempt to make sense of the conflicting data sets and large gaps in our current knowledge.

The book has a strong cosmochemical slant. This is understandable given that meteorites and lunar and terrestrial rock samples provide some of the strongest constraints we have on theories about the origin of the Universe. Still, it is disappointing that certain other important areas are glossed over or abbreviated in the rush to provide a unified theory for the Solar System. For example, it is currently not known how a protoplanetary disk of gas and dust gives rise to mountain-sized 'planetesimals' - bodies that are large enough to have appreciable gravitational fields that can aid further coagulation. The universally favoured planetesimal theory of planet formation is the main theory advocated in Solar System Evolution, yet its shakier foundations are never examined.

Another bone of contention concerns the origin and structure of the giant planets. Some readers may not share Ross Taylor's conviction that the Sun's protoplanetary nebula was driven away by strong solar winds within the first million years of its existence. This is hard to reconcile with the standard model for the formation of giant planets, heartily endorsed here, which begins with the accretion of a ten-Earth-mass solid core, followed by the gradual acquisition of a gaseous envelope. The alternative theory, that Jupiter formed as a result of the gravitational collapse of part of the Sun's protoplanetary disk, is given short shrift, although the evidence for a jovian core is relativelyweak.

The book opens with a sombre account of the achievements of the pioneers of planetary science. This is followed by a dizzying ride through galaxy formation, nucleosynthesis - the formation of atoms through nuclear reactions in the cores of stars - and the origin of stars. We then finally settle down to the serious business of contemplating the Solar System. On the whole, Ross Taylor's writing is fluent and engaging, but he occasionally slips into the annoying habit of using specialized terminology before defining it.

The second half of the book contains a piece-by-piece analysis of each of the main components of the modern Solar System. There is an excellent comparative analysis of Venus, Earth and Mars, which includes a detailed discussion of why Earth alone possessed the conditions necessary to spawn plate tectonics and the formation of a continental crust. In the outer Solar System, the author boldly embraces the idea that Pluto is not a major planet, but rather the leading member of a new breed. The recent discovery of an object in the EdgeworthKuiper belt that has a diameter roughly half that of Pluto's provides timely support for this viewpoint.

A central theme of the book is that the Solar System is unique, the result of a long series of chance events which are unlikely to be repeated elsewhere. Here the author is on firm ground. One of the most striking lessons we havelearned from the discovery of extrasolar planets is that nature prefers variety to consistency when it comes to building planetary systems. Close analogues of the Solar System may turn out to be rare, as are planets that resemble Earth, and this makes our own system all the more fascinating. Despite its occasional shortcomings, the scope of this study is impressive, and this well-researched book deserves a place on the bookshelf of anyone interested in the Solar System and its origins.

John E. Chambers is at the NASA Ames Research Center, MS 245-3, Moffett Field, California 94035, USA.

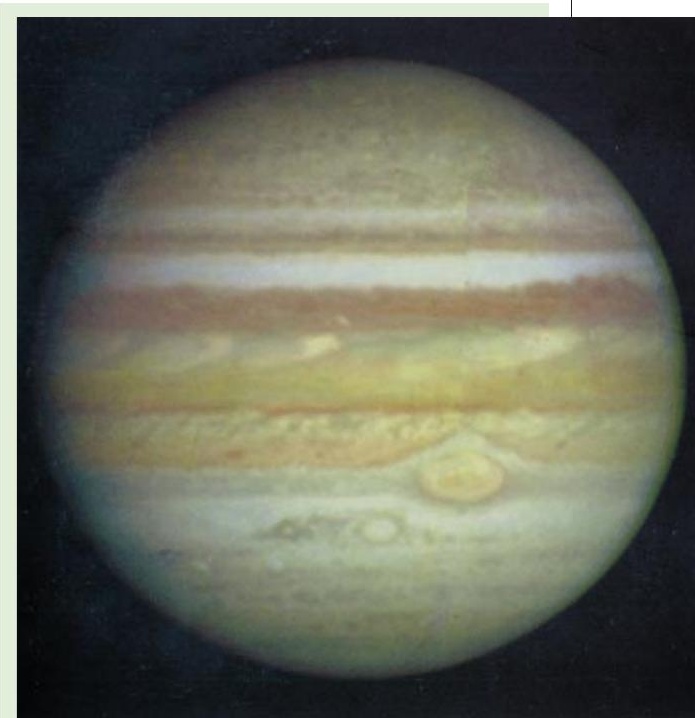

\section{A jaunt through the Solar System}

As its name suggests, The Cambridge Photographic Guide to the Planets by F. W. Taylor (Cambridge University Press, $\$ 49.95, £ 29.95)$ offers a selection of the latest images of the planetary bodies in our Solar System. Starting with Mercury, the book takes the reader on a voyage to the outer edges of our planetary system, encompassing the planets and their respective satellites, plus asteroids and comets along the way. The image shown above, for example, shows Jupiter and was taken by the Hubble Space Telescope. It offers a relatively rare 'true colour' picture of the planet - in most images of Jupiter, the colours are exaggerated to emphasize the contrasts. 